TAMKANG JOURNAL OF MATHEMATICS

Volume 41, Number 4, 349-351, Winter 2010

\title{
RECURSIVE CONSTRUCTION OF MAXIMUM NUMBER OF NON-OVERLAPPING KLEIN 4-GROUPS INSIDE AN ELEMENTARY ABELIAN 2-GROUP
}

\author{
BRETT STEVENS
}

\begin{abstract}
David E. Dobbs produced a recursive construction for large sets of nonoverlapping Klein 4-subgroups inside $\mathbb{Z}_{2}^{n}$. We show that the construction can be used to build the maximum such sets.
\end{abstract}

Let $b_{n}$ denote the cardinality of a maximum set $\mathcal{S}$ of pairwise non-overlapping (except in the identity element) subgroups of $\mathbb{Z}_{2}^{n}$ with each member of $\mathcal{S}$ isomorphic to the Klein 4group. In his paper "On the maximal number of non-overlapping Klein 4-groups inside an elementary Abelian 2-group", David E. Dobbs gives a recursive construction that shows $b_{n+2} \geq 4 b_{n}$ [3]. This question is equivalent to determining the maximum cardinality spread in $\mathrm{PG}(n-1,2)[2,3]$. Although the fact that

$$
b_{2 a}=\frac{2^{2 a}-1}{3} \quad b_{2 a+1}=\frac{2^{2 a+1}-5}{3}
$$

has been known for some time in the finite geometry literature [1, 2], Dobbs's work is interesting because his construction is algorithmic and recursive, it does not depend on the parity of $n$, it achieves the best possible integer multiple and the methods are elementary [3]. I would add two more reasons to this list. The first is that Dobbs's work is very accessible to students in a way that much of the finite geometry literature is not. The second, which is the subject of this note, is that his technique can be used to construct the maximum possible values given in Equation (1). This optimal behaviour complements nicely with the accessibility of the method to a student audience.

In the case of $n$ even the optimality of Dobbs's construction is simple to observe. If the construction is applied to a set $\mathcal{S}$ in $\mathbb{Z}_{2}^{n-2}$ of maximum cardinality $\left(2^{n-2}-1\right) / 3$ then the only group elements not covered by the subgroups of the set $\mathcal{T}$ in $\mathbb{Z}_{2}^{n}$ are $\{(0,0, \ldots, 0,0,1),(0,0, \ldots, 0,1,0),(0,0, \ldots, 0,1,1)\}$ which together with the identity element, form a copy of the Klein 4-group which is disjoint (except for the identity) from all the subgroups in $\mathcal{T}[3]$.

Received October 22, 2009.

2000 Mathematics Subject Classification. 20K01, 05A99.

Key words and phrases. Elementary abelian 2-group, nonoverlapping subgroups, Klein 4-group, maximum partial spread. 
The case where $n=2 a+1$ is odd is more complicated. We first begin with an elementary proof of the upper-bound:

$$
b_{2 a+1} \leq \frac{2^{2 a+1}-5}{3} .
$$

By counting the number of non-identity elements of $\mathbb{Z}_{2}^{n}$, we see that $b_{2 a+1} \leq\left(2^{2 a+1}-2\right) / 3$, so we only need to show that attaining this weaker bound is impossible. If there were a set, $\mathcal{S}$, of non-overlapping Klein 4 -subgroups in $\mathbb{Z}_{2}^{n}$ of cardinality $\left(2^{2 a+1}-2\right) / 3$ then there would be precisely one non-identity element, $x$, not contained in a member of $\mathcal{S}$. Since the sum over all non-identity group elements of $\mathbb{Z}_{2}^{n}$ is the identity and the same is true for the sum of non-identity elements of any Klein 4-subgroup, we see that

$$
\{x\}=\mathbb{Z}_{2}^{n} \backslash\left\{\bigcup_{H \in \mathcal{S}} H\right\},
$$

and thus

$$
\begin{aligned}
x & =\sum_{g \in \mathbb{Z}_{2}^{n}} g-\sum_{g \in \cup_{H \in \mathcal{S} H}} g \\
& =0
\end{aligned}
$$

which is a contradiction.

We now use Dobbs's construction to achieve the maximum cardinality set $\mathcal{S}$. We point out again that the result of our Theorem 1 is known [1]; it is the elementary nature of the proof that is novel.

Theorem 1. Let $n$ be odd. Then

$$
b_{n}=\frac{2^{n}-5}{3} .
$$

Proof. Now when $n=3, b_{3}=1[3]$. This particular set $\mathcal{S}$ has a property that will be maintained by the Dobbs's recursive construction, namely that $\mathcal{S}$ has an element $H_{0}$ such that

$$
\left(\mathbb{Z}_{2}^{n} \backslash\left\{\bigcup_{H \in \mathcal{S}} H\right\}\right) \bigcup H_{0} \cong \mathbb{Z}_{2}^{3} .
$$

Now suppose that $\mathcal{S}$ is a set of non-overlapping Klein 4-subgroups of $\mathbb{Z}_{2}^{n-2}$ of cardinality $\left(2^{n}-5\right) / 3$ with a member $H_{0}=\{0, x, y, z, x+y, x+z, y+z, x+y+z\}$ such that the Property 2 holds. As in the proof of Theorem 1 from [3], we let $G=\mathbb{Z}_{2}^{n}=U \oplus V$, where $U$ denotes the direct sum of the first $n-2$ copies of $\mathbb{Z}_{2}$ and we identify the last two copies of $\mathbb{Z}_{2}$ in $G$ with $V=\{0, a, b, c=a+b\}$.

We apply Dobbs's construction to $\mathcal{S} \backslash\left\{H_{0}\right\}$ to obtain collection $\mathcal{T}^{\prime}$ of Klein 4-subgroups in $G$. The only elements of $G$ not covered by $\mathcal{T}^{\prime}$ are the 31 non-identity elements of $H_{0} \oplus V$. 
We will denote these elements as ordered pairs of elements from $H_{0}$ and $V$ respectively. Let

$\mathcal{T}^{\prime \prime}=\left\{\begin{array}{ll}\{(y, 0),(z, 0)(y+z, 0),(0,0)\}, & \{(y, a),(z, b)(y+z, c),(0,0)\}, \\ \{(y, b),(x+y+c, 0)(x+z, b),(0,0)\}, & \{(y, c),(x+y+z, c),(x+z, 0),(0,0)\}, \\ \{(z, a),(x+y+z, a),(x+y, 0),(0,0)\},\{(z, c),(x+y+z, b),(x+y, a),(0,0)\}, & \\ \{(x+y, b),(x+z, c),(y+z, a),(0,0)\}, & \{(x+y, c),(x+z, a),(y+z, b),(0,0)\}\end{array}\right\}$

and

$$
H_{0}^{\prime}=\{(x, 0),(x, a),(0, a),(0,0)\} .
$$

Now $\mathcal{T}=\mathcal{T}^{\prime} \cup \mathcal{T}^{\prime \prime} \cup\left\{H_{0}\right\}$ is a set of $b_{n}=\left(2^{n}-5\right) / 3$ non-overlapping Klein 4-subgroups in $G$ with member $H_{0}^{\prime}$ such that Property 2 holds.

\section{References}

[1] A. Beutelspacher, Blocking sets and partial spreads in finite projective spaces, Geom. Dedicata, 9(1980), 425-449.

[2] P. Dembowski, Finite geometries, Springer-Verlag, Berlin, 1997. Reprint of the 1968 original.

[3] D. E. Dobbs, On the maximal number of non-overlapping klein 4-groups inside an elementary abelian 2-group, Tamkang J. Math., 40(2009), 113-116.

School of Mathematics and Statistics, Carleton University, 1125 Colonel By Drive, Ottawa ON K1S 5B6, Canada.

E-mail: brett@math.carleton.ca 